\title{
Genome-wide circadian rhythm detection methods: systematic evaluations and practical guidelines
}

Wenwen Mei ${ }^{1,{ }^{*}}$, Zhiwen Jiang ${ }^{1, *}$, Yang Chen $^{2}$, Li Chen $^{3}$, Aziz Sancar ${ }^{4,5, \#}$, Yuchao Jiang $^{1,5,6, \#}$

1 Department of Biostatistics, Gillings School of Global Public Health, University of North Carolina, Chapel Hill, NC 27599, USA.

2 Department of Statistics and Michigan Institute for Data Science, University of Michigan, Ann Arbor, MI 48109, USA.

3 Department of Medicine and Center for Computational Biology and Bioinformatics, Indiana University School of Medicine, Indianapolis, IN 46202, USA.

4 Department of Biochemistry and Biophysics, School of Medicine, University of North Carolina, Chapel Hill, NC 27599, USA.

5 Lineberger Comprehensive Cancer Center, University of North Carolina, Chapel Hill, NC 27599, USA.

6 Department of Genetics, School of Medicine, University of North Carolina, Chapel Hill, NC 27599, USA.

Wenwen Mei is a PhD student in the Department of Biostatistics at the University of North Carolina at Chapel Hill.

Zhiwen Jiang is a MS student in the Department of Biostatistics at the University of North Carolina at Chapel Hill.

Yang Chen is an Assistant Professor in the Department of Statistics and Research Assistant Professor at the Michigan Institute of Data Science at the University of Michigan.

Li Chen is an Assistant Professor in the Dpartment of Medicine and a member of the Center for Computational Biology and Bioinformatics at Indianna University School of Medicine.

Aziz Sancar is the Sarah Graham Kenan Professor of Biochemistry and Biophysics at the University of North Carolina School of Medicine and member of UNC Lineberger Comprehensive Cancer Center.

Yuchao Jiang is an Assistant Professor in the Department of Biostatisrtics and the Department of Genetics at Univerity of North Carolina at Chapel Hill and member of UNC Lineberger Comprehensive Cancer Center.

* These authors contributed equally.

\# To whom correspondence should be addressed. Email: aziz sancar@med.unc.edu; yuchaoj@email.unc.edu. 


\section{ABSTRACT}

2 Circadian rhythms are oscillations of behavior, physiology, and metabolism in many

3 organisms. Recent advancements in omics technology make it possible for genome-wide

4 profiling of circadian rhythms. Here, we conducted a comprehensive analysis of seven

5 existing algorithms commonly used for circadian rhythm detection. Using gold-standard

6 circadian and non-circadian genes, we systematically evaluated the accuracy and

7 reproducibility of the algorithms on empirical datasets generated from various omics

8 platforms under different experimental designs. We also carried out extensive simulation

9 studies to test each algorithm's robustness to key variables, including sampling patterns,

10 replicates, waveforms, signal-to-noise ratios, uneven samplings, and missing values.

11 Furthermore, we examined the distributions of the nominal $p$-values under the null and

12 raised issues with multiple testing corrections using traditional approaches. With our

13 assessment, we provide method selection guidelines for circadian rhythm detection,

14 which are applicable to different types of high-throughput omics data.

16 Key words: biological rhythm; circadian rhythm detection; benchmarking; omics; 17 precision and recall; reproducibility. 


\section{Key points}

- Various methods have been developed for circadian rhythm detection on a genome-wide scale using omics technologies, yet there has not been a comprehensive summary and evaluation of all existing methods to date.

- Using gold-standard circadian and non-circadian genes, we systematically evaluated the accuracy and reproducibility of seven existing algorithms for circadian rhythm detection on empirical datasets generated from various omics platforms.

- We carried out extensive simulation studies to test each algorithm's robustness to key variables, including sampling patterns, replicates, waveforms, signal-to-noise ratios, uneven samplings, and missing values.

- We examined the distributions of the nominal $p$-values under the null and raised issues with multiple testing corrections using the Benjamini-Hochberg procedure due to gene-gene correlation and testing being overly conservative.

- We provide method selection guidelines for circadian rhythm detection, which are applicable to different types of high-throughput omics data. 


\section{BACKGROUND}

35 Circadian rhythms are approximately 24-hour oscillations of behavior, physiology, and

36 metabolism that exist in almost all living organisms ranging from prokaryotes to mammals

37 [1, 2]. Circadian rhythm is regulated by the circadian system, which consists of many 38 "clock-controlled genes" that exhibit oscillatory patterns [1]. These oscillations provide 39 organisms with an adaptive advantage by enabling them to predict and adjust to the 40 variations within their environments [3]. Additionally, and perhaps more importantly, 41 disruptions of circadian rhythms have shown to contribute to numerous diseases, 42 including metabolic disorders, heart disease, and aging [4-7]. It is, therefore, of great 43 importance and interest to perform genome-scale analysis of biological rhythms.

Recent advances in omics technologies, including both microarrays and nextgeneration sequencing, offer appealing platforms to identify circadian genes on a genome-wide scale. These have, indeed, led to the proposal of multifarious methodologies adopted from various fields including mathematics, statistics, astrophysics, etc. The earliest of the selected methods is Lomb-Scargle (LS) periodogram [8], an algorithm adapted from astrophysics that detects oscillations by comparing the data to sinusoidal reference curves of varying periods and phases [9, 10]. ARSER is an algorithm that employs autoregressive spectral estimation to predict periodicity and applies a harmonic regression model to fit the time-series [11]. Unlike the model-based LS and ARSER, JTK_CYCLE is a non-parametric method that detects oscillations by comparing the ranks of the measured values to a set of prespecified symmetric reference curves [3]. Both RAIN and eJTK_CYCLE build on the strengths of JTK_CYCLE: RAIN includes an additional set of asymmetric waveforms and examines the increasing and decreasing portions of the curve separately [12]; eJTK_CYCLE improves JTK_CYCLE by explicitly calculating the null distribution such that it accounts for multiple hypothesis testing and by

59 including non-sinusoidal reference waveforms [13]. Based on the successes of the

60 aforementioned methods, MetaCycle proposes an ensemble framework that integrates

61 results from three different algorithms, LS, ARSER, and JTK_CYCLE [14]. Specifically,

62 MetaCycle detects periodicity using the best of breed methods: its $p$-values are generated

63 using Fisher's method; its periods and phase estimations are integrated using arithmetic 64 and circular means; and a new periodic model, formulated from ordinary least squares 
65 method, is applied to recalculate the amplitude. The most recent method, BIO_CYCLE, 66 is a deep neural network trained on both simulated and empirical circadian and 67 noncircadian time-series [15]. More general information and characteristics of each 68 method are summarized in Table 1.

69 Multiple studies [10, 16, 17] have evaluated the performance of different methods 70 for circadian rhythm detection, showing discrepancies among the methods, whose 71 performances depend on multiple factors including experimental designs, waveforms of 72 interest, etc. However, there has not been, to our best knowledge, a comprehensive 73 summary and evaluation of all existing methods to date. Here, we systematically assess

74 the performance of the seven aforementioned algorithms for circadian rhythm detection: 75 LS, ARSER, JTK_CYCLE, RAIN, eJTK_CYCLE, MetaCycle, and BIO_CYCLE.

Specifically, we demonstrated and benchmarked the algorithms using real datasets with gold-standard circadian and non-circadian genes. All empirical data were generated using the liver tissue from Mus musculus that had undergone two different experimental designs. Under the dark-dark experimental design (24-hour darkness), we focused on using data from gene expression microarrays to assess the accuracy and

81 reproducibility of each algorithm; under the light-dark experimental design (12-hour light

82 followed by 12-hour darkness), we adopted four different next-generation sequencing 83 platforms and explored the robustness of each method in identifying circadian genes.

84 Furthermore, to extend our assessment to non-transcriptomic datasets, we included a 85 proteomic dataset in our evaluation. In addition, we carried out extensive simulation 86 studies to study how key variables, including sampling patterns, replicates, waveforms, 87 signal-to-noise ratios, uneven samplings, missing values, affect the performance of each 88 method. Lastly, we point out the flaw with using the Benjamini-Hochberg procedure to 89 control for false discovery rate. Through these, we offer guidelines on experimental 90 designs as well as best practices and methods of choice to increase the rigor and 91 reproducibility in the analysis of large-scale circadian rhythms. To assist with the 92 comparison of future methods and datasets using our framework, we provide detailed 93 vignettes on applications of existing methods and performance evaluations with source 94 code available at https://github.com/wenwenm183/Circadian Genes Benchmark. 


\section{RESULTS}

97 Performance assessment using empirical datasets with dark-dark design

98 We first adopted three gene expression microarray datasets from Hughes et al. [18],

99 Hughes et al. [19], and Zhang et al. [20]. For all three studies, mouse liver samples were

100 collected in every hour or every two-hour under the dark-dark experimental design for 48

101 hours. We named these three datasets after the first author's last name and the year of

102 publication as Hughes 2009, Hughes 2012, and Zhang 2014, respectively. In addition, we

103 generated a new downsampled dataset from the Hughes 2009 dataset by keeping the

104 even time-points only, and named it "Downsampled Hughes 2009". Refer to Table 2A for

105 details of the data. Figure 1 shows the scaled gene expression levels of four known

106 circadian and four non-circadian genes. The circadian genes, including the well-studied

107 Clock, Cry1, Npas2, and Per1 [10], show oscillatory patterns that can be well reproduced

108 across studies, while the non-circadian genes exhibit only noisy signals.

We set out to apply the seven algorithms to these four datasets to detect significantly cyclic genes and evaluate their performances using 104 circadian [10] and

111113 non-circadian genes [21] from previous studies (Supplementary Table 1). The 112 accuracy of each method in Hughes 2009, Downsampled Hughes 2009, Hughes 2012, 113 and Zhang 2014 was first assayed with the precision and recall rates for each algorithm

114 given three $p$-value thresholds, 0.000005 (Bonferroni), 0.00005, 0.0005, and one $q$-value 115 threshold 0.05 (Benjamini-Hochberg). Due to the tradeoff between sensitivity and 116 specificity, with more relaxed thresholds of significance, the precision rates of all methods 117 decrease while the recall rates increase - the $0.05 q$-value threshold achieves the lowest 118 precision rate yet the highest recall rate for any given method (Figure 2A). While there 119 does not exist a single method that consistently achieves the highest precision or recall 120 rate, JTK_CYCLE and BIO_CYCLE are more effective in controlling for false positives 121 while still detecting true circadian genes. For the other methods, however, there is a much 122 higher variability in precision, especially in the Zhang 2014 dataset (Figure 2A). RAIN and 123 MetaCycle tend to have the highest sensitivity/recall, but this can come with significant 124 sacrifice on precision (Figure 2A).

125 In addition, we find that higher sampling frequency can significantly improve the 126 recall rates of all methods. While MetaCycle and RAIN achieve the apparently higher 
127 recall rate under different thresholds in dataset sampled at a lower frequency (2 h/2 days),

128 all methods, except for LS, produce comparable recall rates when applied to the Hughes

1292009 dataset, which is sampled at $1 \mathrm{~h} / 2$ days (Figure 2A). Notably, when analyzing the

130 three datasets with lower sampling frequencies, LS failed under all circumstances with

131 recall rates less than 0.1 (Figure $2 \mathrm{~A}$ ). This is due to the extreme $p$-value distribution of

132 the method with a spike at one, which we will discuss in more detail under "Correlated

133 multiple testing and non-uniform distribution of $p$-values under the null".

We further computed with the receiver operating characteristic (ROC) curves with

135 a varying threshold on the nominal $p$-values returned by each method (Figure 2B). The

136 area under the curve (AUC) values serve as a joint measure of sensitivity and specificity

137 and are above 0.80 across all benchmark results, suggesting that all methods achieve

138 good sensitivities while controlling for false positive rates. BIO_CYCLE, the deep-

139 learning-based method, achieves the best performance with the highest AUC across all

140 datasets (Figure 2B).

\section{Reproducibility assessment using empirical datasets with dark-dark design}

143 Reproducibility is one of the core principles for any bioinformatic tools and yet it remains

144 a challenge in the field of circadian rhythm detection, which has not been fully explored.

145 To evaluate the reproducibility of the methods, we first compared and contrasted the 146 significantly cyclic genes returned by each method across the four datasets. To make the 147 input dimensions compatible, we selected a total of 7,570 common genes that are shared 148 across datasets and adopted a $q$-value threshold of 0.05 for significance. The Venn 149 diagrams in Figure 3A show the overlapping relationships of the significant genes 150 returned by each method. While the experimental designs are the same and the observed 151 gene expression measurements are highly concordant (Figure 1), significant 152 discrepancies of the calling results are observed. Of the seven benchmarked methods, 153 ARSER resulted in 721 overlapping significant genes, which is the highest. This is 154 followed by RAIN, eJTK_CYCLE, MetaCycle, BIO_CYCLE, JTK_CYCLE, and LS with $155613,528,485,296,204$, and 0 mutually identified positives, respectively. As mentioned 156 previously, LS failed in detecting any significant oscillations for three out of the four 157 datasets. 
To further assess the reproducibility of the methods, we computed the Jaccard index and the Sorensen index to measure the similarities among the results from each method. Details of these metrics are included in the Materials and Methods section. As a result, RAIN achieves one of the highest Jaccard indices for any pair of comparisons and ARSER achieves the highest overall Sorensen index across all datasets (Figure 3B). On the other hand, our results indicate that JTK_CYCLE, eJTK_CYLE, and BIO_CYCLE produce the lowest similarity metrics across all comparisons (Figure 3B).

\section{Performance assessment using empirical datasets with light-dark design}

167 Next, we adopted four datasets that underwent light-dark experimental design using 168 different next-generation sequencing platforms (i.e., RNA-seq [22], Nascent-seq [22], 169 GRO-seq [23], and XR-seq [24]) and named each one after its sequencing protocol (Table 170 2B). The four datasets have much fewer numbers of time-points compared to the datasets 171 from the dark-dark design, yet three of the four datasets have technical replicates (Table 172 2B). More details of the data can be found in the Materials and Methods section. The 173 oscillatory patterns of known circadian genes are apparent and similar among the various 174 sequencing technologies (Figure 4A), indicating good data quality.

ARSER, despite its high reproducibility, cannot handle replicates, and previous studies have shown that data should never be concatenated [17]. Therefore, we focused on assessing the performance of the other six methods. We first examined the distribution of the nominal $p$-values of the 104 gold-standard circadian genes returned by each method, visualized as beehive plots in Figure 4B, where LS is significantly underpowered

180 in the detection of circadian genes compared to the other methods, given any of the 181 sequencing platforms. This result can be attributed to LS's inability to effectively detect

182 circadian rhythms in datasets with low sampling resolution, which is concordant with our 183 previous results. We observe that JTK_CYCLE, RAIN, eJTK_CYCLE, MetaCycle, and 184 BIO_CYCLE can withstand the sparse sampling and result in overall good performance.

To further assess the performance of the methods, we examined the number of 186 significant genes identified by each method with a false discovery rate (FDR) of 0.05. Of 187 the 9,481 mutual genes in the four datasets, LS did not identify any significant genes in 188 any of the datasets. This result aligns with the results from the previous analysis, where 
189 we observed LS as being underpowered. JTK_CYCLE and MetaCycle detected a 190 relatively small number of significant genes by RNA-seq and XR-seq. eJTK_CYCLE 191 identified 2,623 significant genes by RNA-seq, and RAIN and BIO_CYCLE identified 192 2,262 and 1,970 significant genes by XR-seq, respectively. When comparing across 193 different sequencing platforms, we observe that the number of detected significant genes 194 from RNA-seq and XR-seq data is much higher than that of the GRO-seq and Nascent195 seq data. This implicates a potential deficiency in detecting gene expression rhythmicity 196 by measuring nascent transcripts.

197 With the identified significant genes, we further carried out a gene set enrichment 198 analysis using the DAVID web server $[25,26]$ with the default options. Results from the 199 KEGG pathway enrichment analysis are shown in Supplementary Table 2. We find that 200 circadian rhythm is significantly enriched by various algorithms, which are marked with 201 asterisks in Figure 4B. Specifically, we find that of the five methods that were able to 202 identify statistically significant genes from RNA-seq data, all have enriched circadian 203 rhythm pathway. Circadian rhythm is also enriched in the three lists of genes that were 204 identified by eJTK_CYCLE and RAIN as well as two of the three lists of genes identified 205 by BIO_CYCLE.

\section{Performance assessment using empirical proteomic dataset of dark-dark design}

208 To assess performance of the various methods on non-transcriptomic data, we adopted 209 a proteomic dataset of mouse livers under dark-dark experimental design from Robles et. 210 al [27]. Refer to the Materials and Methods section for details. Since this dataset consists 211 of replicates and missing values, only LS, JTK_CYCLE, RAIN, and MetaCycle were 212 directly applicable. eJTK_CYCLE was not included due to its inefficiency in handling 213 random missing values across different genes/proteins. We calculated the number of 214 significant proteins identified by each method using an FDR threshold of 0.05 215 (Supplementary Figure 1A). LS identified the least number of oscillatory proteins. 216 JTK_CYCLE and MetaCycle returned a moderate number of significant proteins. RAIN 217 identified the largest number of oscillatory proteins, 582, exceeding that of other methods 218 by more than 300 . Heatmaps of scaled measurements of oscillatory proteins identified by 219 at least two methods are shown in Supplementary Figure 1B, where the proteins are 
220 ordered based on their inferred phases. With the identified oscillatory proteins, we

221 conducted a gene set enrichment analysis using the DAVID web server. While the results

222 did not indicate that circadian rhythm was significantly enriched by any of the algorithms,

223 KEGG metabolic pathways were significantly enriched by all algorithms but LS

224 (Supplementary Table 3).

225

226 Performance assessment using synthetic datasets

227 To provide guidelines for method selection, we evaluated the performance of the seven 228 methods in detecting circadian rhythm by simulations with known ground truths. 229 Examples of waveforms generated for the simulated datasets are shown in

230 Supplementary Table 4. We generated six groups of simulated datasets to investigate

231 how key factors affect the performance, including sampling patterns, replicates,

232 waveforms, signal-to-noise ratios (SNRs), uneven samplings, and missing values.

233 Supplementary Table 5 outlines the six groups of simulations and we leave the detailed

234 setup in the Materials and Methods section. Within each simulation group, we repeated

235 each assessment with three different sampling frequencies to determine whether

236 increasing sampling frequency may have an effect on the aforementioned factors. The

237 three sampling frequencies include $4 \mathrm{~h} / 1$ day (six time-points), $3 \mathrm{~h} / 1$ day (eight time-

238 points), and $2 \mathrm{~h} / 1$ day (twelve time-points) and the results are shown in Figure 5A, 5B, 239 and 5C, respectively.

\section{Sampling patterns}

241 To determine whether increasing the sampling frequency or lengthening the time-window

242 is more important for each method, we first evaluated the results under the sampling 243 pattern of $4 \mathrm{~h} / 1$ day versus $8 \mathrm{~h} / 2$ days, $3 \mathrm{~h} / 1$ day versus $6 \mathrm{~h} / 2$ days, and $2 \mathrm{~h} / 1$ day versus

$2444 \mathrm{~h} / 2$ days. We did not find strikingly different results within each pair of comparison, 245 indicating that when the total number of data points are fixed, having a denser sampling 246 density and enlarging the sampling time-window tend to have similar impact on 247 performance. However, when we increase the number of data points, the performances 248 of all methods are improved, which is concordant with existing studies [16, 17]. 249 BIO_CYCLE generally outperforms the other methods, especially in datasets with lower 
250 sampling frequency and shorter time-window, while JTK_CYCLE is the most sensitive to

251 fewer observations.

252 Replicates

253 To investigate the trade-off between replicates and sampling frequency, we compared 254 the results of higher sampling frequency without replicates to those of lower sampling 255 frequency with replicates. We first compared the dataset sampled at $4 \mathrm{~h} / 1$ day $\mathrm{X} 1$ to the 256 dataset sampled at 8 h/1 day X2. LS, JTK_CYCLE, RAIN, eJTK_CYCLE, and MetaCycle 257 show better performance with replicates, while BIO_CYCLE performs significantly better 258 on densely sampled datasets without replicates. Similar results are seen when we applied 259 the methods to the dataset at $3 \mathrm{~h} / 1$ day without replicates and the dataset at $6 \mathrm{~h} / 1$ day 260 with replicates. As expected, further increasing the sampling resolution offsets the 261 existing preferences that the methods have for inclusion of replicates or higher sampling 262 density.

\section{Waveforms}

264 Supplementary Table 4 outlines the different types of periodic waveforms that we 265 generated in silico in three broad categories: stationary, non-stationary, and asymmetric 266 ones. Through our simulations, we find that all of the algorithms perform the best in 267 detecting non-stationary waveforms. Additionally, all methods, with the exception of 268 eJTK_CYCLE, perform better on stationary waveforms, compared to asymmetric 269 waveforms. eJTK_CYCLE and RAIN are the top two methods for identifying asymmetric 270 waveforms, which are expected due to their design. This is followed by LS, BIO_CYCLE,

271 MetaCycle, and ARSER. JTK_CYCLE is the least effective in identifying asymmetric 272 waveforms regardless of sampling frequency.

\section{Signal-to-noise ratios (SNRs)}

274 To test the effects of different noise levels on method performance, we generated various 275 datasets with signal-to-noise ratios of 3, 2, 1, and 0.5. For all methods, our results suggest 276 that the larger the SNRs, the higher the accuracy, as expected. LS, MetaCycle, and 277 BIO_CYCLE are overall the most robust to noises regardless of sampling frequency, 278 while JTK_CYCLE has the poorest performance given high noise levels. 


\section{Uneven samplings}

280 To understand how well the methods deal with uneven samplings, we focus on the results

281 of datasets with one or more uneven time-points. Our results suggest that BIO_CYCLE

282 and LS/MetaCycle outperform the other two compatible methods. Under a sparse 283 sampling design, RAIN and eJTK_CYCLE suffer significantly from an increasing number

284 of uneven samplings; a dense sampling design, on the other hand, rescues the 285 aforementioned methods.

\section{Missing values}

287 We generated datasets that contain 1\%, 5\%, and 10\% missing data, and benchmarked 288 the four methods that allow missing values. The performances of eJTK_CYCLE and RAIN 289 degrade with an increasing proportion of missing values, while the performances of LS, 290 JTK_CYCLE, and MetaCycle are comparably invariant, especially under dense sampling 291 design. We note that eJTK_CYCLE does not handle missing values efficiently, unless the 292 same sampling time points are missing across all genes, which reduces to uneven 293 sampling. When there is not a shared missing pattern across different genes, the dataset 294 needs to be split into multiple uneven sampling cases, and eJTK_CYCLE needs to be 295 applied separately, followed by results integration. Note that BIO_CYCLE can be applied 296 to datasets with missing values only if there are replicates and the missingness only 297 pertains to part of the replicates. We therefore did not include it in the benchmark.

\section{Computational efficiency}

299 Last but not least, we evaluated the computational efficiency across all benchmarked 300 methods. For dataset with low sampling resolution, the execution times among the 301 methods are approximately the same (Supplementary Table 6). However, when analyzing 302 data of larger sizes, RAIN requires significantly more time compared to the other methods. 303 The running time for LS, ARSER, and BIO_CYCLE does not change much with varying 304 sampling frequency. The running time for MetaCycle, which integrates results from LS, 305 JTK_CYCLE, and ARSER, is calculated as the total running time of the three methods.

307 Correlated multiple testing and non-uniform distribution of $\boldsymbol{p}$-values under the null 308 To detect circadian rhythm across thousands of genes, multiple hypothesis testing 309 corrections are needed [28]. A common FDR threshold of 0.05 is recommended by most 
310 methods and adjusted $p$-values ( $q$-values) are returned by all methods except for RAIN.

311 In the previous sections, we adopted both Bonferroni and Benjamini-Hochberg

312 procedures for corrections. Here, we more carefully examine such procedures and point

313 out a potential drawback resulted from both correlated multiple testing and non-uniform

314 distributions of the nominal $p$-values under the null. We started with the observed

315 expression measurements from the Hughes 2009 dataset and generated a "null" dataset

316 by randomly permuting the time labels for each gene (Figure 6A). Such permutations not

317 only deplete each gene's rhythmic signals but also disrupts any gene-gene correlations

318 as observed in the raw data, which are high between genes in the same pathways (Figure

319 6B). As such, all genes upon permutations are under the true null and additionally all

320 gene-level testing is independent.

$321 \quad$ Figure $6 \mathrm{C}$ shows the distributions of nominal $p$-values for each method when

322 applied to the dataset before and after permutation. The "U-shaped" histograms of the $p$ -

323 values for LS, JTK_CYCLE, MetaCycle, and RAIN using the original data indicate that

324 there is dependence among the variables in the data. This violates the underlying

325 assumption of uniformity and raises a red flag for using Bonferroni or FDR for error control

326 [28]. A few methods have been developed for $p$-value adjustment when the tests are

327 correlated [29-31] and such issue has been specifically pointed out by Hutchison and

328 Dinner [32] for circadian rhythm detection.

329 We further applied the methods to the permuted data without gene-gene 330 correlations. The hypothesis testing by LS, JTK_CYCLE, RAIN, and MetaCycle are still 331 overly conservative, while the testing procedures for ARSER and BIO_CYCLE are biased 332 with an overabundance of $p$-values around 0.3 and 0.1 , respectively. eJTK_CYCLE 333 empirically calculates the null distribution of the $p$-values via permutations and its 334 enhanced version, booteJTK, speeds up this calculation by approximating the null 335 distribution of the Kendall's tau using a Gamma distribution [33]. This indeed leads to a $336 p$-value distribution closest to the null. However, neither eJTK_CYCLE nor booteJTK 337 handles missing values efficiently, as explained previously. As a summary, there is still 338 room for method development to yield $p$-values that better match the underlying 339 assumption of a uniformly distributed $p$-values under the null. 


\section{DISCUSSION}

342 Here, we propose a benchmark framework to systematically evaluate the performance of 343 seven circadian rhythm detection methods, using high-throughput omics data. The 344 empirical datasets that we adopted in this paper were from microarray [18-20] and RNA345 seq [22] to measure gene expression, Nascent-seq [22] and GRO-seq [23] to measure 346 nascent RNA, and XR-seq [24] to measure transcription-coupled repair. While these 347 omics data were generated from different platforms, they focus on directly or indirectly 348 profiling transcription. It has been well studied that biological rhythm goes beyond the 349 transcriptomic transcript-level oscillations [34]. For example, post-translational protein 350 acetylation has been linked to circadian rhythm via mass spectrometry [35, 36]. Moreover,

351 it has been shown that a large number of metabolites and proteins exhibit circadian 352 oscillations [27, 37, 38]. The methods and the evaluation procedures are not limited to 353 transcriptomic studies, but can also be applied to acetylomic, metabolomic, and proteomic 354 experiments.

Given the assessment results from both simulations and empirical dataset anaylsis, as well as literature review of the seven methods, we have summarized the strengths and weaknesses of each method in Table 3. In general, LS, RAIN, eJTK_CYCLE, and 358 MetaCycle are more versatile in that they can be applied to datasets with replicates, 359 uneven samplings, or missing values. eJTK_CYCLE and BIO_CYCLE generally 360 outperform the other methods under most situations except for handling missing values. 361 On the other hand, JTK is sensitive to high noise levels and low sampling resolutions, 362 and LS cannot detect any significant genes when sampling resolution is lower than $2 \mathrm{~h} / 2$ 363 days with an FDR threshold of 0.05 . The best detection algorithm depends on 364 experimental designs and characteristics of the input data. Therefore, we have created 365 two decision trees, one for low sampling resolution and the other for high sampling 366 resolution, that outline the recommended method(s) under different scenarios 367 (Supplementary Figure 2).

Recent advances of high-throughput technologies enable circadian rhythm 369 detection on the genome-wide scale. As with all genomic data, the multi-time-point omics 370 data for circadian rhythm detection bear both technical and biological variability, which 371 can bias the analysis if not properly accounted. Data normalization and batch effect 
372 correction are crucial to remove technical biases and artifacts [39]. Cross-subject 373 variability in rhythmic profiles, especially for human subjects, is a non-negligible source 374 of genetic variation that needs to be adjusted [14]. This is especially important in the case375 control setting where multiple subjects are involved. While we did not particularly focus 376 on differential analysis since it is outside the scope of this paper, a few methods, including 377 LimoRhyde [40] and DODR [12] have been made available for differential rhythmicity 378 analysis under different conditions.

379 Increasingly more circadian omics data are being made available through existing 380 studies and databases [34, 41]. We showed, from our empirical studies, that the rhythmic 381 signals can be well recapitulated across different studies and/or different platforms 382 (Figure 1, Figure 4A). Meta-analysis and multi-omics data integration remain an open383 ended question in circadian rhythm detection [42]. In addition, transfer learning has been 384 applied to multiple genomic research domains in genomics [43] - to borrow information 385 and to transfer knowledge from existing data deposited in public repositories remain one 386 of the future directions. Similarly, across different methods, an ensemble framework, as 387 implemented by MetaCycle, can potentially boost performance. However, as we have 388 pointed out earlier, the instability issue needs to be addressed, especially when multiple 389 drastically distinct results are to be integrated.

$390 \quad$ To our best knowledge, all existing studies for circadian rhythm detection resort to 391 bulk-tissue omics data, which characterize an averaged profile across different cell types 392 in a tissue. The inherent heterogeneity can bias the analysis with reduced power and/or 393 inflated FDR. Single-cell sequencing circumvents the averaging artifacts associated with 394 traditional bulk population data and has seen rapid technological developments over the 395 past few years. To assess the feasibility of single-cell circadian rhythm detection, we in 396 silico generated single-cell RNA sequencing profiles by downsampling bulk RNA-seq 397 read counts. Gold-standard circadian and noncircadian genes were used to calculate the 398 associated AUC values (Supplementary Table 7). All methods suffer from low sequencing 399 depth - a characteristic of the single-cell data. With the decreasing cost and the 400 increasing popularity of single-cell omics techniques, to profile circadian rhythmicity at the 401 cellular level and to disentangle within tissue heterogeneity with regard to biological 402 rhythm can be of great impact. 


\section{MATERIALS AND METHODS}

\section{Empirical transcriptomic datasets}

406 Three datasets under the dark-dark experimental design including Hughes 2009 [18], 407 Hughes 2012 [19], and Zhang 2014 [20] were downloaded from GEO, and all used 408 microarrays to profile gene expressions (Table 2A). Additionally, we obtained four 409 datasets under the light-dark experimental design from the different sequencing platforms, 410 including Nascent-sequencing (Nascent-seq) [22], RNA-sequencing (RNA-seq) [22], 411 Global Run-On sequencing (GRO-seq) [23], and eXcision Repair-sequencing (XR-seq) 412 [24] (Table 2B). Nascent-seq sequence transcribed RNAs, obtained from the nuclei 413 without formation of the 3' end [44]. GRO-seq measures nascent RNAs by mapping, 414 characterizing, and evaluating transcriptionally engaged polymerase [45]. GRO-seq and 415 Nascent-seq differ from traditional RNA-seq, in which the reads map to predominantly 416 introns, while RNA-seq mainly assays exons [44]. XR-seq profiles DNA excision repair 417 on the genome-wide scale with single-nucleotide resolution [46]. Here, we focus on XR418 seq data from the transcribed strand only - it has been shown that the transcription419 coupled repair from the transcribed strand is positively correlated with expression [47].

For quality control, we removed genes that had constant gene expression

421 measurements in all datasets and further removed genes with more than half zero gene 422 expression values in the light-dark datasets. In cases where multiple probes got mapped 423 to the same RefSeq loci, we averaged the gene expression of the probes using the limma 424 package [48], available in Bioconductor. For data normalization, robust multi-array 425 average (RMA) [49] and genechip RMA (GC-RMA) [50] were used to normalize the array 426 data; transcript per million (TPM) and reads per kilobase per million reads (RPKM) [51] 427 were used to normalize the transcriptomic sequencing data. We scaled the normalized 428 data within each gene to make them compatible for visualization only, as shown in Figure 4291 and Figure 4A.

\section{Empirical proteomic dataset}

432 A proteomic dataset of Mus musculus liver tissues from Robles et. al [27] was adopted to 433 detect oscillatory proteins. Mouse liver samples were collected from a total of 64 mice 
434 that were released into constant darkness for one day after being entrained to a 12-12 435 hour light-dark schedule for 10 days. Four mice were sacrificed every 3 hours for 2 days. 436 Then, in vivo Stable Isotope Labeling by Amino acids in Cell culture (SILAC) [52, 53] in 437 combination with mass spectrometry was performed to profile the proteome. For each 438 time point, equal amount of protein liver extracts from the four mice were mixed together 439 with equal amount of protein lysates, collected in anti-phase, from the liver samples of 440 two SILAC mice. The pooled protein extracts were measured with Orbitrap mass 441 spectrometer. The protein abundance was calculated by taking the ratio of the signal for 442 the mice and the signal for the heavy SILAC mix. After assessing quantification values, a 443 total of 3,132 proteins remained for downstream circadian rhythm analysis.

\section{Downsampled RNA-seq dataset}

446 We generated several downsampled RNA-seq datasets from the original RNA-seq 447 dataset under the light-dark design to assess the robustness of the various methods to 448 low sequencing depths. We obtained the raw sequencing data from GEO, performed read 449 alignment to the mouse reference genome (mm10) using STAR [54], carried out quality 450 control procedures on the aligned reads, and obtained integer-valued read counts using 451 featureCounts [55]. We then generated downsampled RNA-seq data by multinomial 452 sampling with index $5 \mathrm{~K}, 10 \mathrm{~K}, 50 \mathrm{~K}, 100 \mathrm{~K}$, and $500 \mathrm{~K}$, and gene-specific probability 453 parameters calculated from the raw data. RPKM was used to normalize the downsampled 454 RNA-seq read counts, followed by circadian rhythm detection.

\section{Evaluation metrics}

457 To evaluate the performance of the benchmarked methods, we adopted a list of 104 458 circadian [10] and 113 non-circadian genes [21] in mouse liver as positive and negative 459 controls, respectively. See Supplementary Table 1 for a full list of these gold-standard 460 genes. With these gold-standard genes, we calculated metrics including the precision and 461 recall rates given a $p$-value or $q$-value significance threshold (Figure $2 A$ ). We further 462 calculated the AUC values of the ROC curves, as joint measures of sensitivity and 463 specificity (Figure 2B). 
To assess the reproducibility of each method, we compared the results from the four dark-dark datasets by calculating the number of overlapping genes, as well as the Jaccard and Sorensen index as metrics for similarity (Figure 3). Venn diagrams are used to display the number of overlapping cycling genes identified across different datasets by each method. The Jaccard index measures the pairwise similarities of the significant genes detected between each pair of datasets. Let $A_{i}$ and $A_{j}$ be the set of significant genes from dataset $i$ and $j$. The Jaccard similarity index is defined as

$$
J\left(A_{i}, A_{j}\right)=\frac{\left|A_{i} \cap A_{j}\right|}{\left|A_{i} \cup A_{j}\right|}
$$

472 The Sorensen Index is used to characterize similarity across all datasets [56]:

$$
S\left(A_{i}, A_{j}, A_{k}, \ldots\right)=\frac{T}{T-1}\left(\frac{\sum_{i<j}\left|A_{i} \cap A_{j}\right|-\sum_{i<j<k}\left|A_{i} \cap A_{j} \cap A_{k}\right|+\sum_{i<j<k<l}\left|A_{i} \cap A_{j} \cap A_{k} \cap A_{l}\right|-\cdots}{\sum_{i}\left|A_{i}\right|}\right)
$$

where $T$ is the number of sets compared. Larger number of overlapping genes and larger Jaccard/Sorensen index values indicate higher reproducibility of the methods.

\section{Simulation setup}

478 Each simulated dataset consists of 6,000 circadian and 6,000 non-circadian gene profiles. Stationary circadian profiles with a period of 24 hours are used in each simulation group, as outlined below. Note that when running the methods, we set the period range from 20 to $28 \mathrm{~h}$ for all methods except for eJTK_CYCLE and JTK_CYCLE, which either has a 482 fixed period of $24 \mathrm{~h}$ or adjusts the period on the fly. The amplitude of the waveforms is sampled from a uniform distribution between 1 and 6; the phase shift is sampled from a uniform distribution between 0 and $24 \mathrm{~h}$; and the noise term is sampled from a standard normal distribution. Flat waveforms are used to generate non-circadian profiles in all simulation groups except for testing against non-stationary waveforms where linear lines are used.

We first aimed to investigate whether higher sampling frequency or longer sampling time-window is more beneficial for each method. In this simulation group, we generated two datasets with different sampling frequencies and sampling time-windows. With six time-points, we generated one dataset at $4 \mathrm{~h} / 1$ day and another at $8 \mathrm{~h} / 2$ days; with eight time-points, we generated one dataset at $3 \mathrm{~h} / 1$ day and another at $6 \mathrm{~h} / 2$ days; with 12 time-points, we generated one dataset at $2 \mathrm{~h} / 1$ day and another at $4 \mathrm{~h} / 2$ days. 
Next, we assessed whether the inclusion of replicates can offset the effect of low sampling frequency in methods' ability of detecting oscillations. Replicates are defined as multiple measurements taken at the same time-point. Specifically, we generated two datasets consisting of the same number of observations, with or without replicates: one at $4 \mathrm{~h} / 1$ day $\mathrm{X} 1$ and the other at $8 \mathrm{~h} / 1$ day $\mathrm{X} 2$. The sampling design of the other two pairs of datasets are 3 h/1 day X1 v.s. 6 h/1 day X2, and 2 h/1 day X1 v.s. 4 h/1 day X2.

Since biological rhythms can take on various waveforms, we generated three types of waveforms via simulation: stationary, non-stationary, and asymmetric curves. Supplementary Table 4 includes models that we adopted in silico to generate the corresponding waveforms. Specifically, the stationary waveforms include cosine, cosine 2 , and cosine peak curves; the non-stationary waveforms include cosine damp, trend exponential, and trend linear curves; the asymmetric subgroup consists of only the sawtooth waveform. We assessed the performance of the methods in identifying each category of the circadian waveforms.

The next three groups of simulations aimed to determine which methods are more robust to different levels of signal-to-noise ratios, uneven samplings, and missing values. Specifically, we generated four datasets with SNRs of 0.5, 1, 2, and 3. Signal-to-noise ratio is defined by taking the ratio of the empirical variance of cosine function and the variance of the noise, the latter of which is fixed at one. Uneven samplings are defined as designs whose time-points are not equally spaced. To investigate the effect of uneven samplings on performance, we generated datasets with one, two, or four uneven samplings. With six time-points, datasets with four uneven samplings cannot be generated as it would only have two time-points. For missing data, we generated three levels of missing data $(1 \%, 5 \%$, and $10 \%)$ at three fixed, randomly selected time-points. days, and $4 \mathrm{~h} / 2$ days to compute the execution times for each method. We seek to identify

520 the differences in computational efficiency among the methods and to explore the effect 521 of increasing sampling resolution on the execution time. Each dataset consists of a total 522 of 6,000 genes. All execution times are reported by running on a Macbook Pro (15-inch, 523 2019) with $2.3 \mathrm{GHz} 8$-Core Intel Core i9 and $16 \mathrm{~GB}$ memory. 
525 DATA AND SOFTWARE AVAILABILITY

526 MetaCycle is an open-source $\mathrm{R}$ package available at

527 https://github.com/gangwug/MetaCycle and is also used for individual analysis for LS, 528 JTK_CYCLE, and ARSER. RAIN is a Bioconductor $R$ package available at 529 https://bioconductor.org/packages/rain/. eJTK_CYCLE was downloaded from 530 https://github.com/alan/hutchison/empirical-JTK CYCLE-with-asymmetry. BIO_CYCLE 531 was downloaded from http://circadiomics.igb.uci.edu/BIO CYCLE. All empirical datasets 532 were downloaded from the NCBI Gene Expression Omnibus

533 (https://www.ncbi.nlm.nih.gov/geo/). The accession numbers for dark-dark datasets are 534 GSE11923, GSE30411, and GSE54652, respectively. The accession numbers for light535 dark datasets are GSE59486, GSE36872, GSE36871 and GSE109938, respectively. The 536 proteomic dataset was downloaded from the BioStudies database with accession number 537 S-EPMC3879213.

\section{ACKNOWLEDGEMENTS}

540 This work was supported by NIH Grants R35 GM118102 (to A.S.), R01 ES027255 (to 541 A.S.), P01 CA142538 (to Y.J.), UL1 TR002489 (to Y.J.), and a pilot award from the UNC 542 Computational Medicine Program (to Y.J.). We thank the Sancar Lab members and Dr. 543 John Hogenesch for helpful discussions and feedback.

\section{REFERENCES}

546 1. Li J, Grant GR, Hogenesch JB, Hughes ME: Considerations for RNA-seq analysis of circadian rhythms. Methods Enzymol 2015, 551:349-367.

2. Panda S, Antoch MP, Miller BH, Su Al, Schook AB, Straume M, Schultz PG, Kay SA,

3. Hughes ME, Hogenesch JB, Kornacker K: JTK_CYCLE: an efficient nonparametric

4. Asher G, Sassone-Corsi P: Time for food: the intimate interplay between nutrition, metabolism, and the circadian clock. Cell 2015, 161:84-92.

556 5. Partch CL, Green CB, Takahashi JS: Molecular architecture of the mammalian circadian 
558 6. Roenneberg T, Merrow M: The Circadian Clock and Human Health. Curr Biol 2016, 26:R432-443.

$5607 . \quad$ Levi F, Schibler U: Circadian rhythms: mechanisms and therapeutic implications. Annu Rev Pharmacol Toxicol 2007, 47:593-628.

8. Glynn EF, Chen J, Mushegian AR: Detecting periodic patterns in unevenly spaced gene expression time series using Lomb-Scargle periodograms. Bioinformatics 2006, 22:310316.

9. Wijnen $\mathrm{H}$, Naef $\mathrm{F}$, Young MW: Molecular and statistical tools for circadian transcript profiling. Methods Enzymol 2005, 393:341-365.

10. Wu G, Zhu J, Yu J, Zhou L, Huang JZ, Zhang Z: Evaluation of five methods for genomewide circadian gene identification. J Biol Rhythms 2014, 29:231-242.

11. Yang R, Su Z: Analyzing circadian expression data by harmonic regression based on autoregressive spectral estimation. Bioinformatics 2010, 26:i168-174.

12. Thaben PF, Westermark PO: Detecting rhythms in time series with RAIN. J Biol Rhythms 2014, 29:391-400.

13. Hutchison AL, Maienschein-Cline M, Chiang AH, Tabei SM, Gudjonson H, Bahroos N, Allada R, Dinner AR: Improved statistical methods enable greater sensitivity in rhythm detection for genome-wide data. PLoS Comput Biol 2015, 11:e1004094.

14. Wu G, Anafi RC, Hughes ME, Kornacker K, Hogenesch JB: MetaCycle: an integrated R package to evaluate periodicity in large scale data. Bioinformatics 2016, 32:3351-3353.

15. Agostinelli F, Ceglia N, Shahbaba B, Sassone-Corsi P, Baldi P: What time is it? Deep

16. Deckard A, Anafi RC, Hogenesch JB, Haase SB, Harer J: Design and analysis of large-scale

17. Hughes ME, Abruzzi KC, Allada R, Anafi R, Arpat AB, Asher G, Baldi P, de Bekker C, Bell-

18. Hughes ME, DiTacchio L, Hayes KR, Vollmers C, Pulivarthy S, Baggs JE, Panda S, Hogenesch biological rhythm studies: a comparison of algorithms for detecting periodic signals in biological data. Bioinformatics 2013, 29:3174-3180.

19. Hughes ME, Hong HK, Chong JL, Indacochea AA, Lee SS, Han M, Takahashi JS, Hogenesch JB: Brain-specific rescue of Clock reveals system-driven transcriptional rhythms in peripheral tissue. PLoS Genet 2012, 8:e1002835.

591 20. Zhang R, Lahens NF, Ballance HI, Hughes ME, Hogenesch JB: A circadian gene expression atlas in mammals: implications for biology and medicine. Proc Natl Acad Sci U S A 2014, 111:16219-16224.

594 21. Wu G, Zhu J, He F, Wang W, Hu S, Yu J: Gene and genome parameters of mammalian 
596 22. Menet JS, Rodriguez J, Abruzzi KC, Rosbash M: Nascent-Seq reveals novel features of mouse circadian transcriptional regulation. Elife 2012, 1:e00011.

598 23. Fang B, Everett LJ, Jager J, Briggs E, Armour SM, Feng D, Roy A, Gerhart-Hines Z, Sun Z, Lazar MA: Circadian enhancers coordinate multiple phases of rhythmic gene transcription in vivo. Cell 2014, 159:1140-1152.

601 24. Yang Y, Adebali O, Wu G, Selby CP, Chiou YY, Rashid N, Hu J, Hogenesch JB, Sancar A: Cisplatin-DNA adduct repair of transcribed genes is controlled by two circadian programs in mouse tissues. Proc Natl Acad Sci U S A 2018, 115:E4777-E4785.

604 25. Huang da W, Sherman BT, Lempicki RA: Systematic and integrative analysis of large gene lists using DAVID bioinformatics resources. Nat Protoc 2009, 4:44-57.

26. Huang da W, Sherman BT, Lempicki RA: Bioinformatics enrichment tools: paths toward the comprehensive functional analysis of large gene lists. Nucleic Acids Res 2009, 37:113.

27. Robles MS, Cox J, Mann M: In-vivo quantitative proteomics reveals a key contribution of post-transcriptional mechanisms to the circadian regulation of liver metabolism. PLoS Genet 2014, 10:e1004047.

612 28. Storey JD, Tibshirani R: Statistical significance for genomewide studies. Proc Natl Acad Sci U S A 2003, 100:9440-9445.

614 29. Li J, Ji L: Adjusting multiple testing in multilocus analyses using the eigenvalues of a correlation matrix. Heredity (Edinb) 2005, 95:221-227.

616 30. Conneely KN, Boehnke M: So many correlated tests, so little time! Rapid adjustment of P values for multiple correlated tests. Am J Hum Genet 2007, 81:1158-1168.

31. Schwartzman A, Lin X: The effect of correlation in false discovery rate estimation.

620 32. Hutchison AL, Dinner AR: Correcting for Dependent P-values in Rhythm Detection.

33. Hutchison AL, Allada R, Dinner AR: Bootstrapping and Empirical Bayes Methods Improve

34. Ceglia N, Liu Y, Chen S, Agostinelli F, Eckel-Mahan K, Sassone-Corsi P, Baldi P: CircadiOmics: circadian omic web portal. Nucleic Acids Res 2018, 46:W157-W162.

626 35. Masri S, Patel VR, Eckel-Mahan KL, Peleg S, Forne I, Ladurner AG, Baldi P, Imhof A, Sassone-Corsi $\mathrm{P}$ : Circadian acetylome reveals regulation of mitochondrial metabolic pathways. Proc Natl Acad Sci U S A 2013, 110:3339-3344.

629 36. Mauvoisin D, Atger F, Dayon L, Nunez Galindo A, Wang J, Martin E, Da Silva L, Montoliu I, 630 Collino S, Martin FP, et al: Circadian and Feeding Rhythms Orchestrate the Diurnal Liver Acetylome. Cell Rep 2017, 20:1729-1743.

632 37. Dallmann R, Viola AU, Tarokh L, Cajochen C, Brown SA: The human circadian metabolome. 
634 38. Feng D, Lazar MA: Clocks, metabolism, and the epigenome. Mol Cell 2012, 47:158-167.

39. Jiang $Y$, Wang R, Urrutia E, Anastopoulos IN, Nathanson KL, Zhang NR: CODEX2: fullspectrum copy number variation detection by high-throughput DNA sequencing. Genome Biol 2018, 19:202.

40. Singer JM, Fu DY, Hughey JJ: Simphony: simulating large-scale, rhythmic data. PeerJ 2019, 7:e6985.

41. Li X, Shi L, Zhang K, Wei W, Liu Q, Mao F, Li J, Cai W, Chen H, Teng H, et al: CirGRDB: a database for the genome-wide deciphering circadian genes and regulators. Nucleic Acids Res 2018, 46:D64-D70.

42. Patel VR, Eckel-Mahan K, Sassone-Corsi P, Baldi P: CircadiOmics: integrating circadian genomics, transcriptomics, proteomics and metabolomics. Nat Methods 2012, 9:772773.

43. Eraslan G, Avsec Z, Gagneur J, Theis FJ: Deep learning: new computational modelling techniques for genomics. Nat Rev Genet 2019, 20:389-403.

44. Trott AJ, Menet JS: Regulation of circadian clock transcriptional output by CLOCK:BMAL1. PLoS Genet 2018, 14:e1007156.

45. Core LJ, Waterfall JJ, Lis JT: Nascent RNA sequencing reveals widespread pausing and divergent initiation at human promoters. Science 2008, 322:1845-1848.

46. Hu J, Adar S, Selby CP, Lieb JD, Sancar A: Genome-wide analysis of human global and transcription-coupled excision repair of UV damage at single-nucleotide resolution. Genes Dev 2015, 29:948-960.

47. Yimit A, Adebali O, Sancar A, Jiang Y: Differential damage and repair of DNA-adducts induced by anti-cancer drug cisplatin across mouse organs. Nat Commun 2019, 10:309.

48. Ritchie ME, Phipson B, Wu D, Hu Y, Law CW, Shi W, Smyth GK: limma powers differential expression analyses for RNA-sequencing and microarray studies. Nucleic Acids Res 2015, 43:e47.

49. Irizarry RA, Hobbs B, Collin F, Beazer-Barclay YD, Antonellis KJ, Scherf U, Speed TP: Exploration, normalization, and summaries of high density oligonucleotide array probe level data. Biostatistics 2003, 4:249-264.

50. Wu Z, Irizarry RA, Gentleman R, Martinez-Murillo F, Spencer F: A Model-Based Background Adjustment for Oligonucleotide Expression Arrays. Journal of the American Statistical Association 2004, 99:909-917.

51. Conesa A, Madrigal P, Tarazona S, Gomez-Cabrero D, Cervera A, McPherson A, Szczesniak MW, Gaffney DJ, Elo LL, Zhang X, Mortazavi A: A survey of best practices for RNA-seq data analysis. Genome Biol 2016, 17:13.

52. Geiger T, Cox J, Ostasiewicz P, Wisniewski JR, Mann M: Super-SILAC mix for quantitative proteomics of human tumor tissue. Nat Methods 2010, 7:383-385. 
671 53. Gouw JW, Krijgsveld J, Heck AJ: Quantitative proteomics by metabolic labeling of model organisms. Mol Cell Proteomics 2010, 9:11-24.

673 54. Dobin A, Davis CA, Schlesinger F, Drenkow J, Zaleski C, Jha S, Batut P, Chaisson M, Gingeras TR: STAR: ultrafast universal RNA-seq aligner. Bioinformatics 2013, 29:15-21.

55. Liao Y, Smyth GK, Shi W: featureCounts: an efficient general purpose program for

677 56. Diserud OH, Odegaard F: A multiple-site similarity measure. Biol Lett 2007, 3:20-22.

\section{FIGURE \& TABLE LEGENDS}

680 Figure 1. Examples of circadian and non-circadian benchmark gene expressions 681 among three datasets with dark-dark experimental design. Scaled gene expressions 682 from selected (A) circadian genes including Clock, Cry1, Npas2, and Per1 and (B) non683 circadian genes including Utp6, Mtf1, Cln3, Abcd4.

Figure 2. Evaluation of seven methods by precision, recall rates and ROC curves. value threshold of 0.05 (FDR threshold) are adopted for each of the seven methods applied to the four dark-dark empirical datasets. A more relaxed threshold results in a higher recall rate, with FDR being the most sensitive, yet this also leads to a higher number of false positives with a lower precision rate. (B) ROC curves and AUC values using gold-standard circadian and non-circadian genes. Each method is evaluated across

692 four dark-dark empirical datasets. Sensitivity and specificity are calculated using the 693 nominal $p$-values by each method with varying threshold. BIO_CYCLE returns the highest 694 AUC.

696 Figure 3. Evaluation of method reproducibility. (A) Venn diagrams display the number 697 of cyclic genes that are significant by each method among the four dark-dark datasets. 698 (B) Jaccard index and the Sorensen index are used as metrics for reproducibility for each 699 method across the four datasets with the same experimental design.

701 Figure 4. Circadian rhythm detection under light-dark experimental design by GRO702 seq, Nascent-seq, RNA-seq, and XR-seq. (A) Gene-specific measurements of nascent 
703 RNA, RNA, and transcription-coupled repair of four circadian benchmark genes, Clock,

704 Npas2, Cry1, and Per1 by four different sequencing platforms. The solid and dotted lines

705 are used for the first and second replicates respectively. (B) Beehive plots of negative log

$706 p$-values of base 10 of circadian genes as positive controls. The number of significant

707 genes detected by each method with an FDR threshold of 0.05 are shown in parenthesis.

708 The asterisks denote significant GO enrichments of circadian rhythm pathway. The 709 nominal $p$-values by JTK_CYCLE, MetaCycle, and BIO_CYCLE are the most significant, 710 while LS and RAIN tend to be underpowered. ARS is not included in the analysis because 711 it cannot be applied to datasets with replicates.

712

713 Figure 5. Performance assessment via simulation studies. Seven circadian rhythm

714 detection methods are evaluated under different experimental designs to explore how 715 sampling patterns, replicates, waveforms, signal-to-noise ratios (SNRs), uneven 716 samplings, and missing values affect performance. Simulations under each design are 717 carried out with different sampling frequencies: (A) 4 h/1 day, (B) 3 h/1 day, and (C) 2 h/1 718 day. AUC values calculated from ground truths are used as metrics.

Figure 6. Existing methods return non-uniformly distributed $p$-values under the null, 721 partially due to non-independent testing due to gene-gene correlations. (A) Gene 722 expression values for the benchmark circadian gene Cry1 before and after random 723 permutations of the time labels. (B) Heatmaps of pairwise correlation coefficients among 724 the top 200 highly variable genes from the Hughes 2009 dataset. The top illustrates the 725 gene-gene correlation coefficients calculated from raw data input, and the bottom shows 726 the gene-gene correlations after permutation. (C) The distributions of nominal $p$-values 727 for each method when applied to the dataset before and after permutation. Gene-gene 728 correlations, which are accounted for by eJTK_CYCLE, partially lead to the systematic 729 deviations from the null distributions. The hypothesis testing by LS, JTK_CYCLE, RAIN, 730 and MetaCycle are overly conservative, while ARSER's and BIO_CYCLE's testing 731 procedures are biased with an overabundance of $p$-values around 0.3 and 0.1 , 732 respectively, under the null. 
734 Table 1. Summary of seven existing methods for circadian rhythm detection. ${ }^{a}$

735 BIO_CYCLE can be applied to datasets with missing values only if there are replicates

736 and the missingness only pertains to part of the replicates.

Table 2. High-throughput mouse liver datasets adopted for circadian rhythm detection. (A) Dark-dark experimental design. (B) Light-dark experimental design.

Table 3. Pros and cons of circadian rhythm detection methods.

SUPPLEMENTARY FIGURE \& TABLE LEGENDS

744 Supplementary Table 1. Circadian and non-circadian genes in Mus muculus liver

745 as gold standard. The 104 circadian gene list is extracted from Supplementary Table 4

746 in Wu et al. Wu G, Zhu J, Yu J, Zhou L, Huang JZ and Zhang Z [10] and the 113 non-

747 circadian gene list is obtained from Supplementary Table 2 in Wu et al. Wu G, Zhu J, He

748 F, Wang W, Hu S and Yu J [21].

Supplementary Table 2. Pathway enrichment analysis of significantly cyclic genes

751 from the light-dark datasets. Functional annotations (KEGG pathway mapping) of the

752 significant genes ( $q$-values $\leq 0.05)$ are carried out using the the DAVID Bioinformatics 753 Resources (https://david.ncifcrf.gov/). The list only contains significantly enriched 754 pathways with a 0.05 cutoff of the $p$-values adjusted by Benjamini Hochberg.

Supplementary Table 3. Pathway enrichment analysis of significantly cyclic proteins. Functional annotations (KEGG pathway mapping) of the significant proteins ( $q$ values $\leq$ 0.05) are carried out using the the DAVID Bioinformatics Resources (https://david.ncifcrf.gov/). The list only contains significantly enriched pathways with a

7600.05 cutoff of the $p$-values adjusted by Benjamini Hochberg. KEGG metabolic pathways 761 were enriched by all three methods.

763 Supplementary Table 4. In silico generated periodic v.s. non-periodic gene profiles.

764 Three types of periodic waveforms are included: stationary, non-stationary, and 
asymmetric. The stationary and non-stationary subgroups consist of three forms of cosine curves. The asymmetric subgroup consists of a saw-tooth waveform. Flat or linear lines are adopted to generate non-periodic waveforms. The waveforms shown are constructed without noise. 'Amp', 'pha', and 'per' represent amplitude, phase and period, respectively.

Supplementary Table 5. Details of simulation setup and parameters used to in silico periodic and 6,000 non-periodic gene profiles. All simulated waveforms have a period length of 24, a phase shift that is uniformly distributed between 0 and 24, and a noise term with standard normal distribution. The amplitude is uniformly distributed between 1 and 6 for all groups except when testing for different signal-to-noise ratios (SNRs), which we define as the ratios of the empirical variances of the cosine function and the variances of the noise. Non-periodic profiles are sampled from a flat/linear function. " $X 1$ " indicates no replicate and "X 2 " indicates two replicates.

\section{Supplementary Table 6. Evaluation of computational efficiency with different}

sampling rates. Each method is run on a dataset with a total of 6,000 genes. All programs are run on a Macbook Pro (15-inch, 2019) with 2.3 GHz 8-Core Intel Core i9 and 16 GB memory. Running time for MetaCycle is the sum of the runing time for LS, ARSER, and JTK_CYCLE. Running time for BIO_CYCLE does not include the time used

785 to fit the deep neural network.

Supplementary Table 7. Performance assessment of downsampled RNA-seq data.

788 AUC values of downsampled RNA-seq datasets with varying sequencing depths were 789 calculated. Existing methods suffer from low sequencing depths. The performance of

790 RAIN exceeds that of all other methods in all sequencing depths with an exception at 5K, 791 due to its large number of significant genes detected in general. BIO_CYCLE consistently 792 ranks the lowest at all but the highest sequencing depth. The performances of LS, 793 JTK_CYCLE, eJTK_CYCLE, and MetaCycle are comparable. 
795 Supplementary Figure 1. Circadian rhythm detection of Mus musculus liver

796 protemoic dataset. (A) Bar plot of the number of significant proteins detected by each

797 method using an FDR threshold of 0.05 . Only methods that are able to handle both

798 replicates and missing values were applied and evaluated. (B) Heatmap of scaled

799 measurements of oscillatory proteins identified by at least two methods. Proteins (rows)

800 are ordered based on their inferred phases.

802 Supplementary Figure 2. Decision tree as user guidance on method selection. The

803 decision tree has decision rules for sampling resolutions, uneven samplings, replicates, 804 and missing values. 
(A)

Cryl

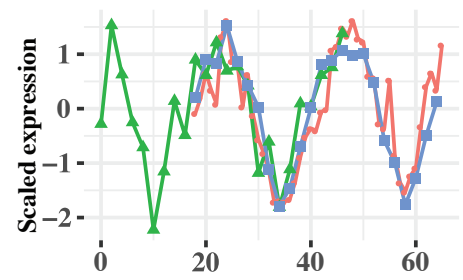

(B)

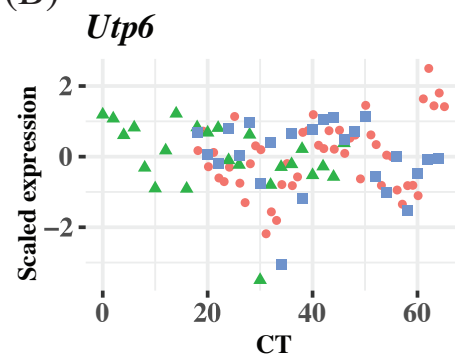

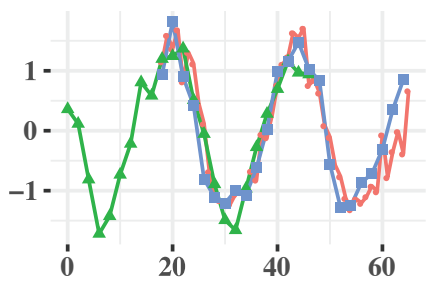

\section{Mtf1}

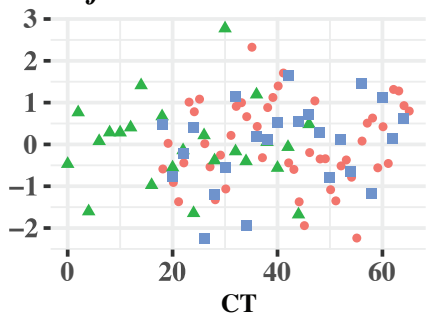

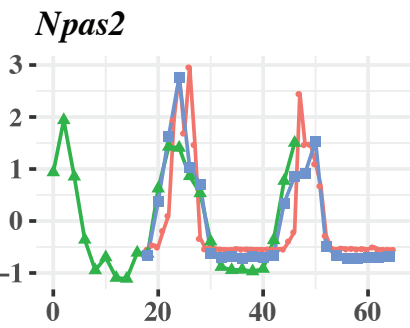
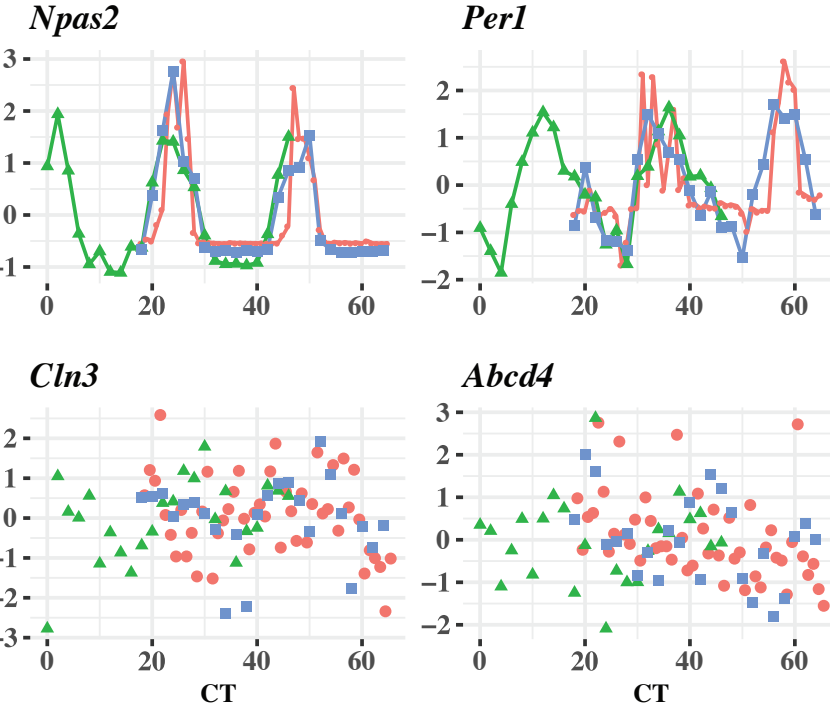

Study

- Hughes 2009

Abcd4

$\triangle$ Hughes 2012

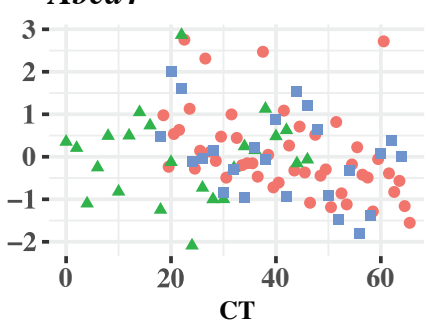

- Zhang 2014

Figure 1 
bioRxiv preprint doi: https://doi.org/10.1101/2020.04.04.024729; this version posted May 15, 2020. The copyright holder for this preprint (which was not certified by peer review) is the author/funder, who has granted bioRxiv a license to display the preprint in perpetuity. It is made available under aCC-BY-NC 4.0 International license.

(A)

Hughes 2009
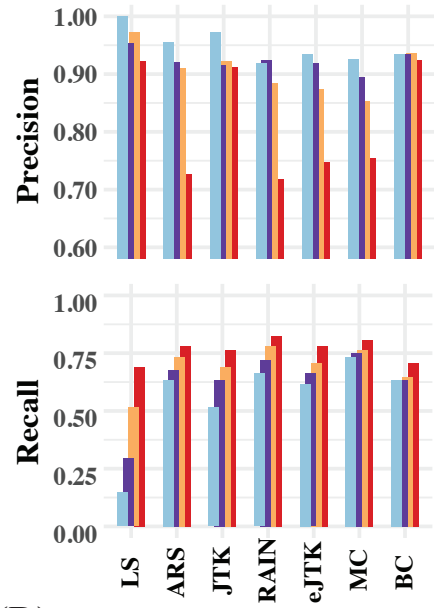

(B)

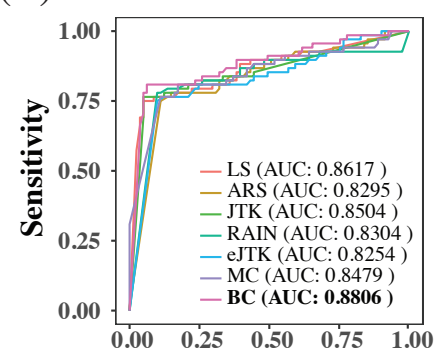

Downsampled
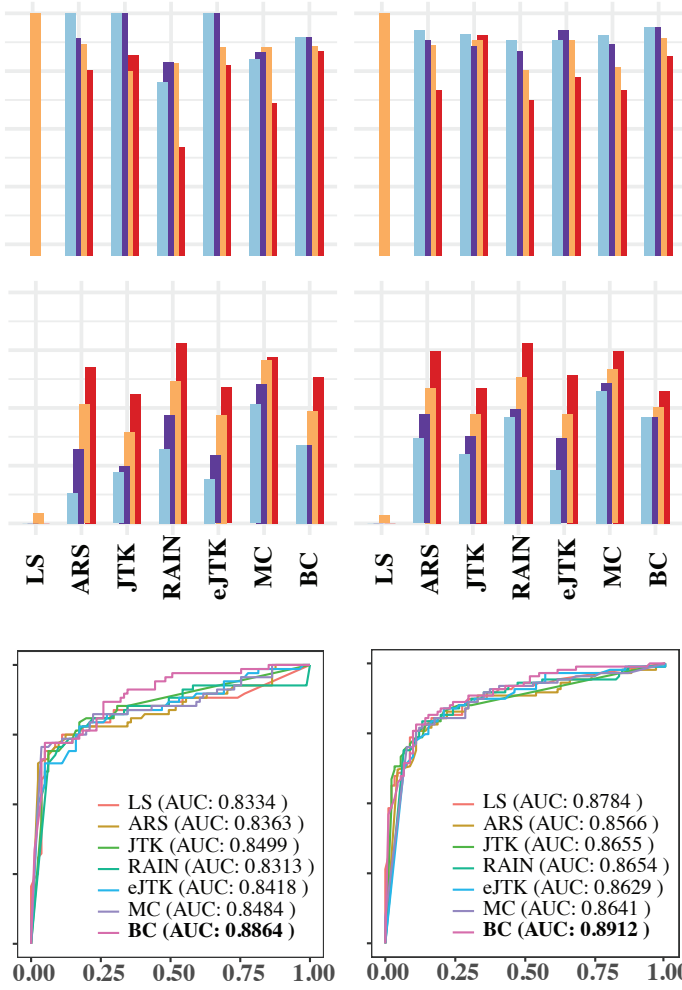

Hughes 2012

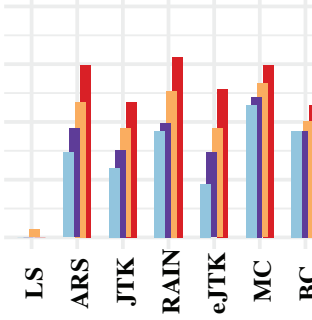

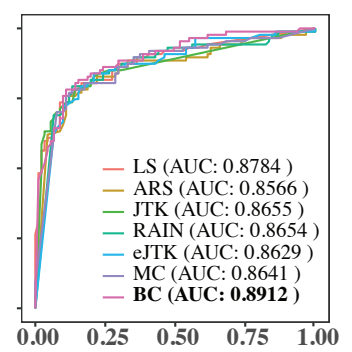

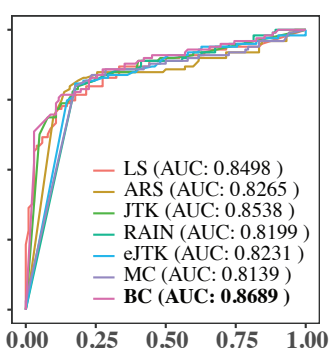

Zhang 2014
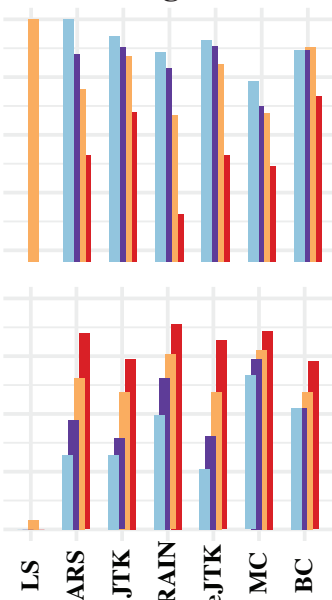

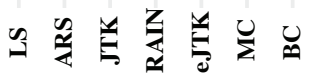

1 - Specificity

Figure 2 
(A)

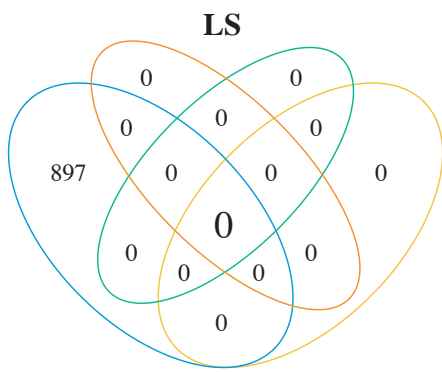

eJTK

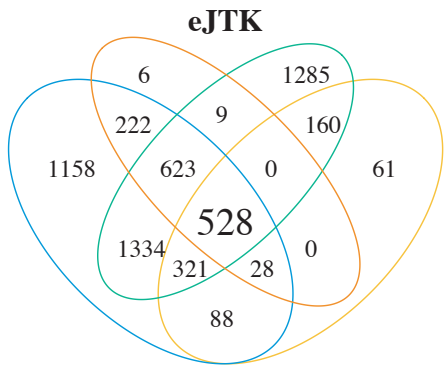

(B)

\begin{tabular}{|c|c|c|c|}
\hline $\begin{array}{c}\text { Hughes } \\
2009\end{array}$ & 0 & 0 & 0 \\
\hline & $\begin{array}{l}\text { Down- } \\
\text { sampled }\end{array}$ & NA & NA \\
\hline & & $\begin{array}{l}\text { Hughes } \\
2012\end{array}$ & NA \\
\hline \multicolumn{3}{|c|}{$\begin{array}{l}\text { LS } \\
\text { Sorensen Index = } 0\end{array}$} & $\begin{array}{l}\text { Zhang } \\
2014\end{array}$ \\
\hline
\end{tabular}

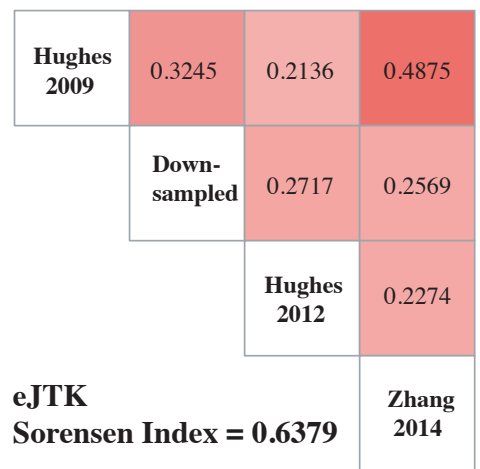

ARS

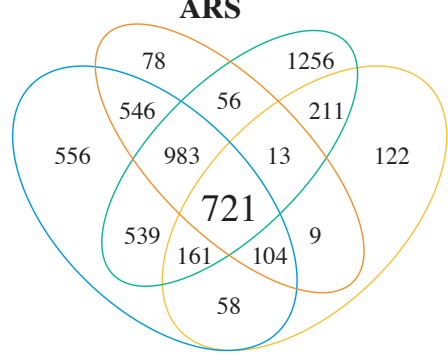

MC
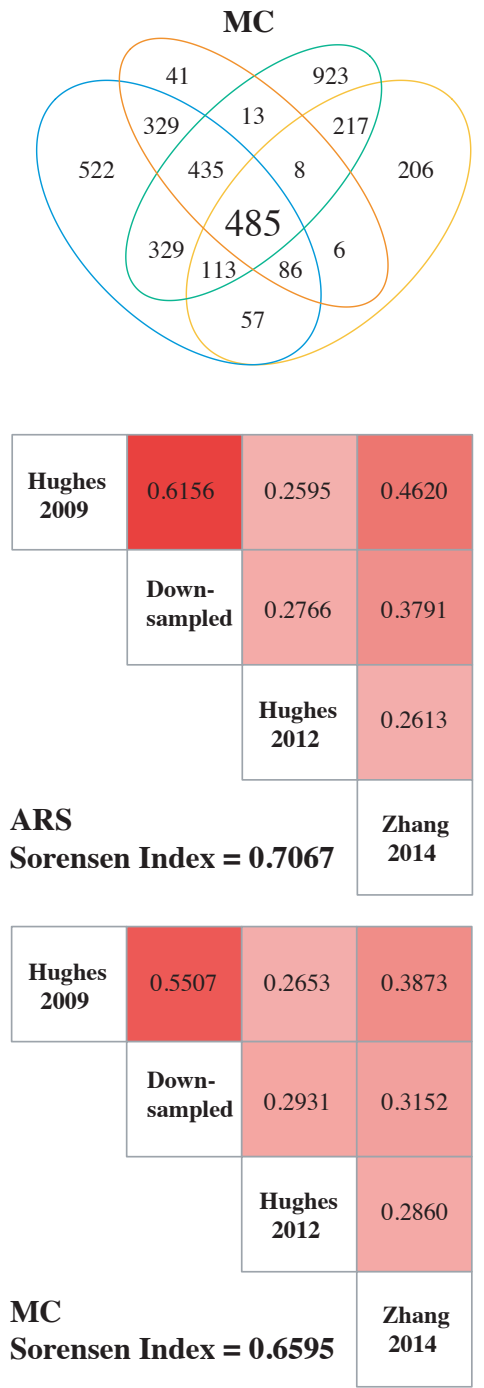

JTK

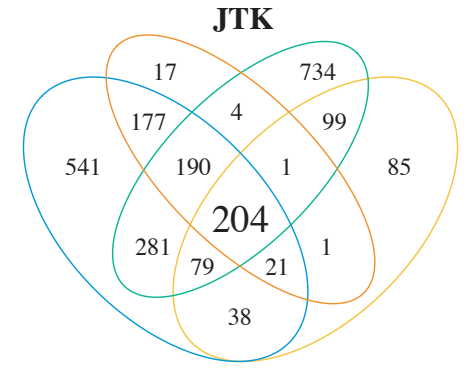

BC

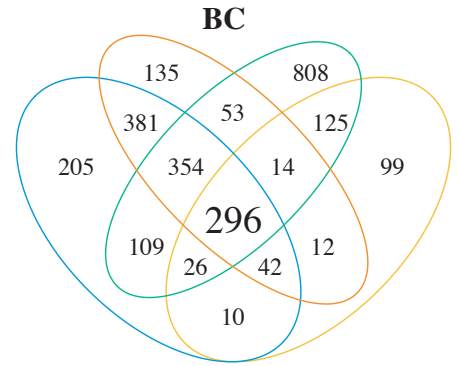

Hughes 2009

Downsampled Hughes 2009

Hughes 2012

Zhang 2014

RAIN
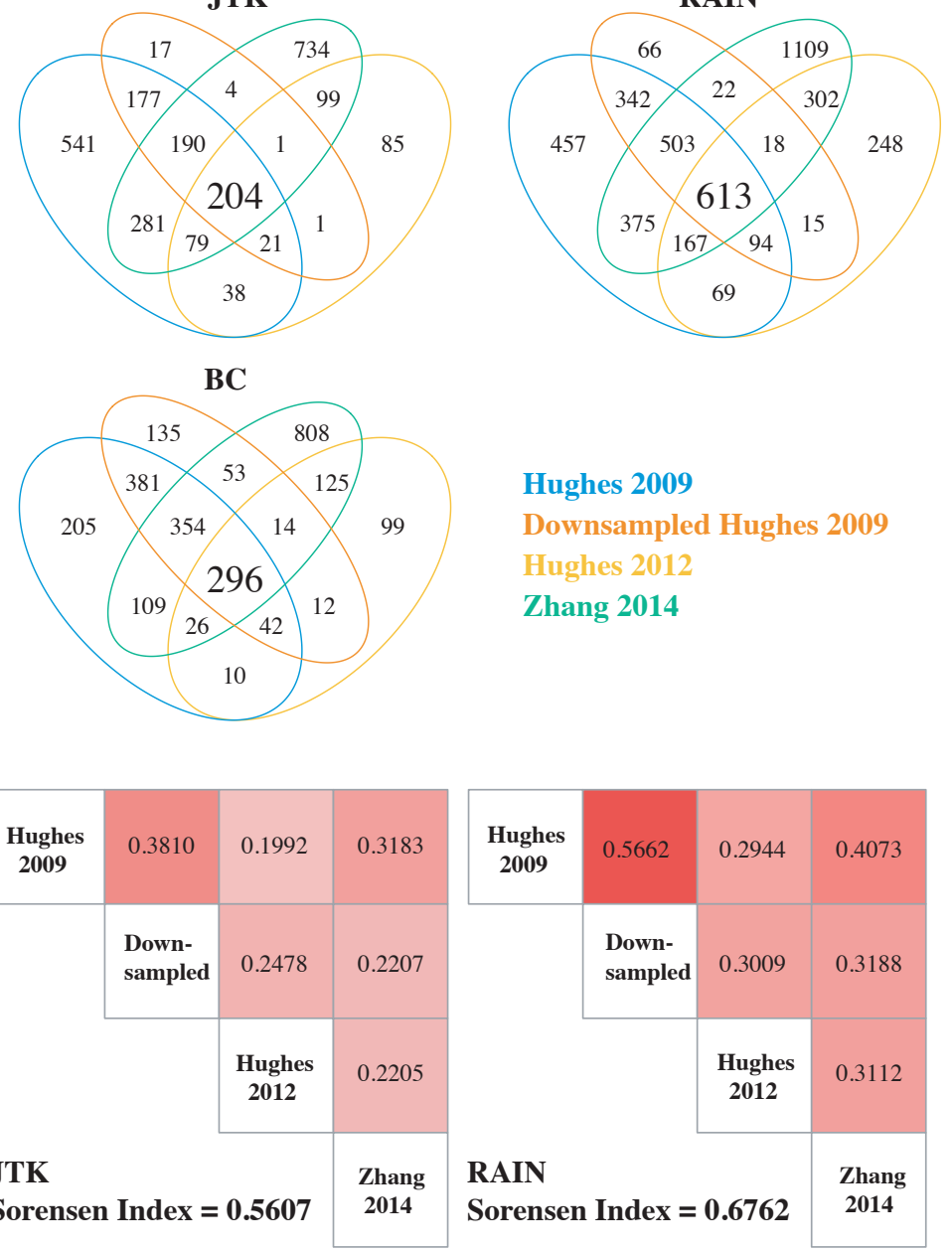
bioRxiv preprint doi: https://doi.org/10.1101/2020.04.04.024729; this version posted May 15, 2020. The copyright holder for this preprint (which

was not certified by peer review) is the author/funder, who has granted bioRxiv a license to display the preprint in perpetuity. It is made available under aCC-BY-NC 4.0 International license.

(A)
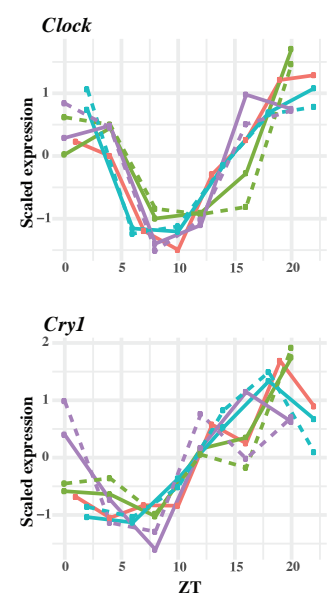

Npas2

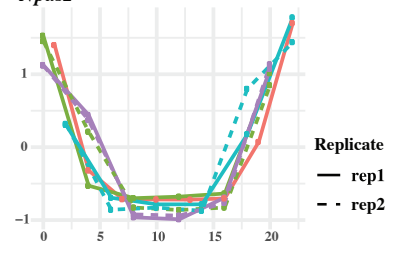

Platform

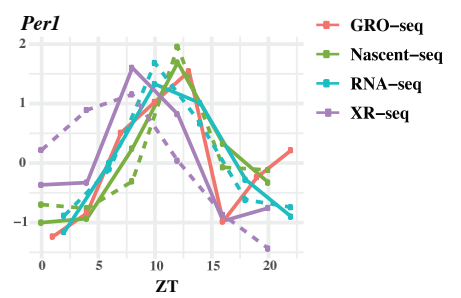

(B)
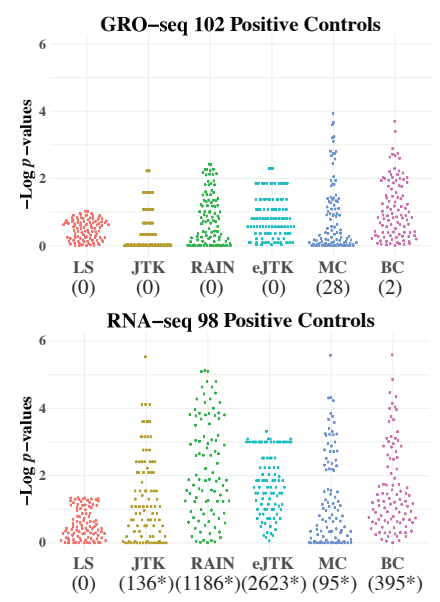

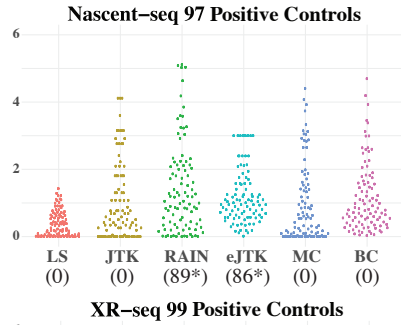

6

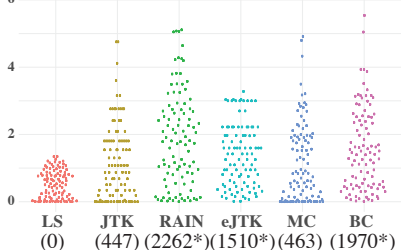


bioRxiv preprint doi: https://doi.org/10.1101/2020.04.04.024729; this version posted May 15, 2020. The copyright holder for this preprint (which

was not certified by peer review) is the author/funder, who has granted bioRxiv a license to display the preprint in perpetuity. It is made available under aCC-BY-NC 4.0 International license.

(A)
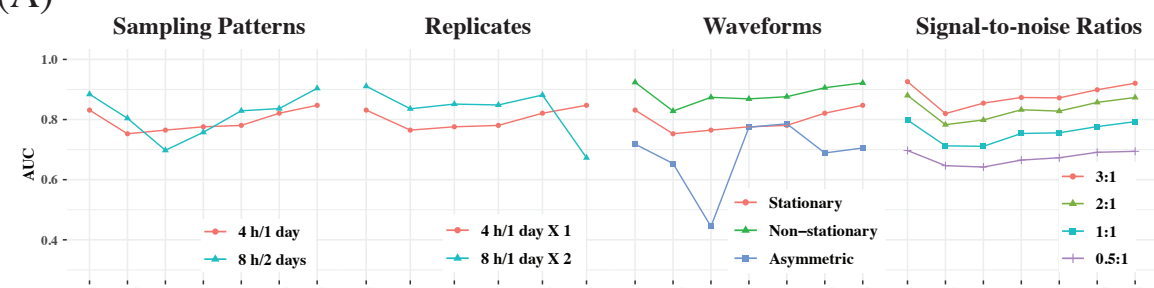

Uneven Samplings

Missing Values

(B)

is ís JTK RAiN elTK MiC

ís ARS JT'K R'IN

L'S ARS JTK RÁIN eJTK MiC B'C
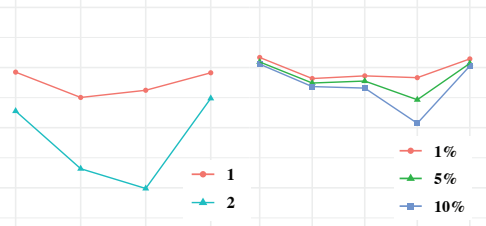

$=5 \%$
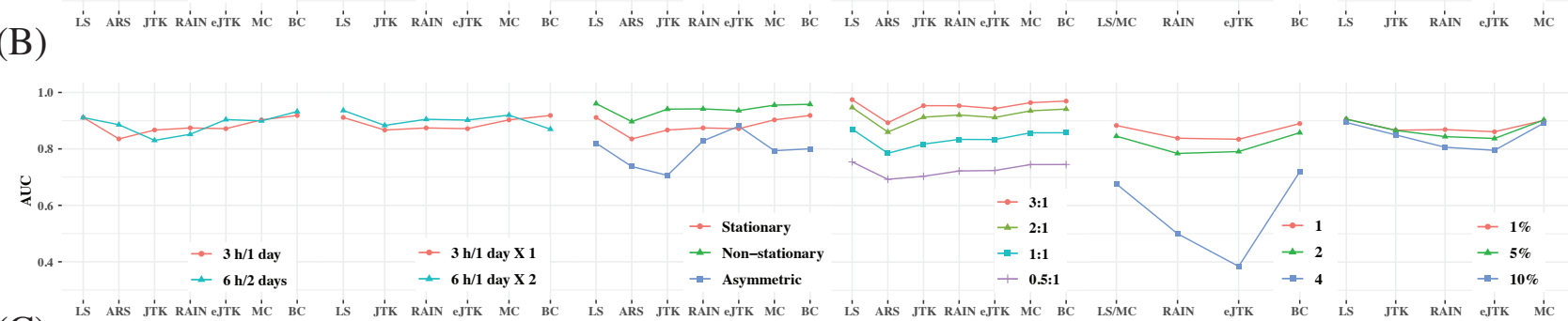

(C)
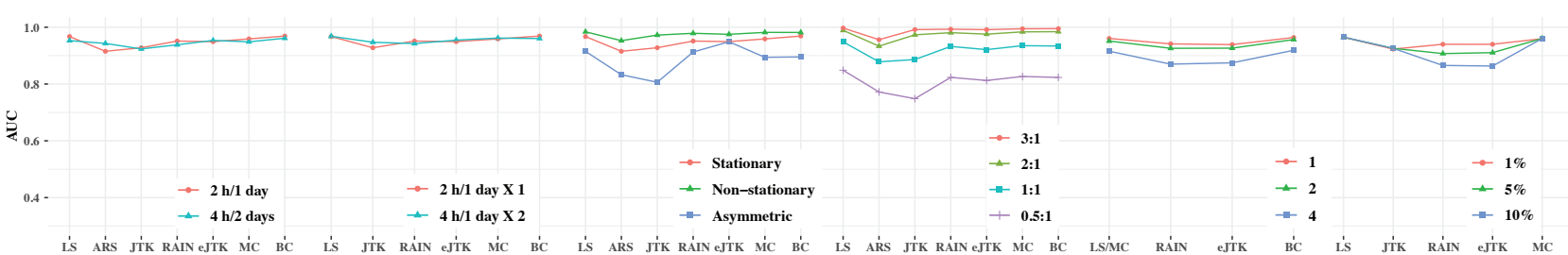

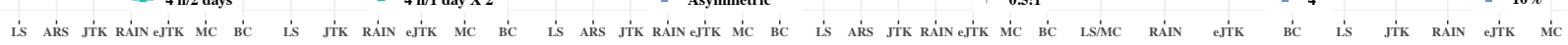


bioRxiv preprint doi: https://doi.org/10.1101/2020.04.04.024729; this version posted May 15, 2020. The copyright holder for this preprint (which

was not certified by peer review) is the author/funder, who has granted bioRxiv a license to display the preprint in perpetuity. It is made available under aCC-BY-NC 4.0 International license.

(A)
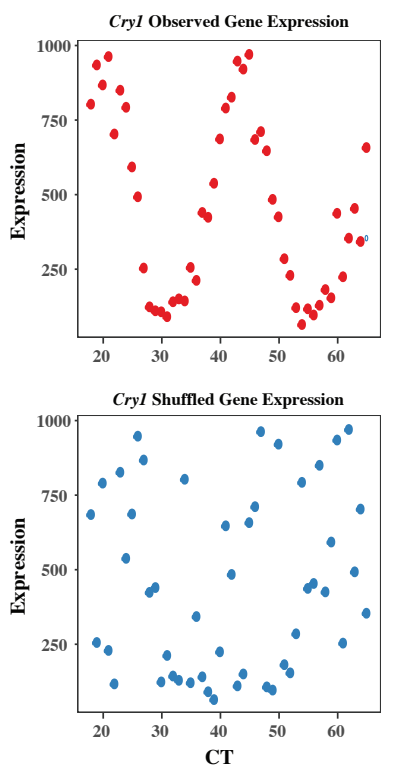

(B)

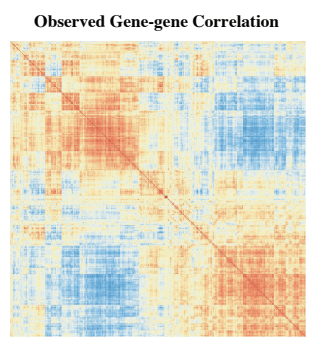

Shuffled Gene-gene Correlation

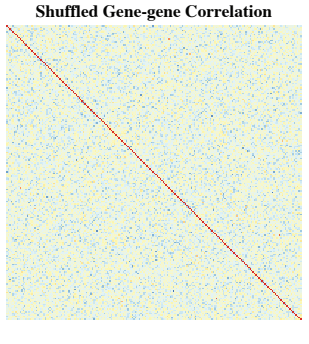

(C) Distribution of Nominal $p$-values (Hughes 2009)
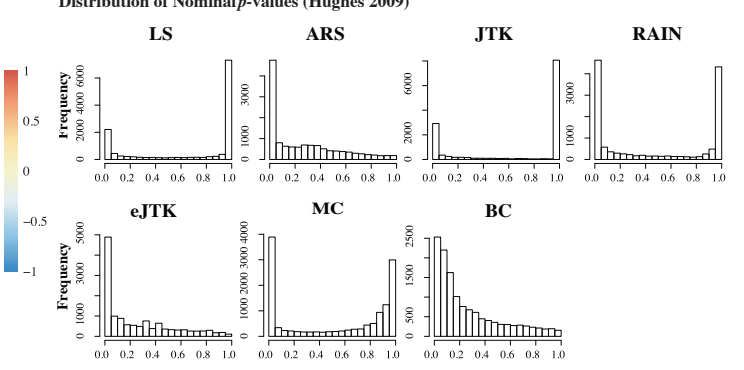

Distribution of Nominal $p$-values (Shuffled Hughes 2009)

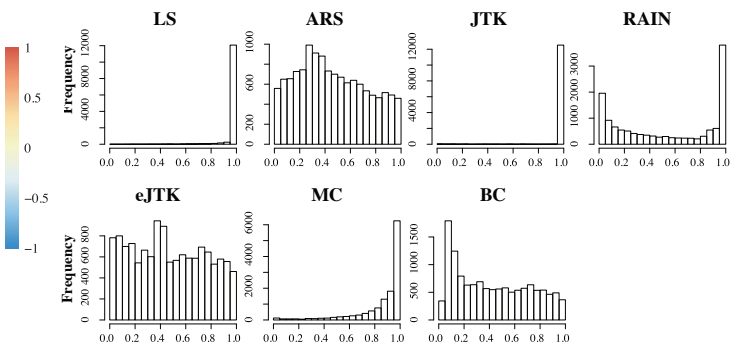


bioRxiv preprint doi: https://doi.org/10.1101/2020.04.04.024729; this version posted May 15, 2020. The copyright holder for this preprint (which

was not certified by peer review) is the author/funder, who has granted bioRxiv a license to display the preprint in perpetuity. It is made available under aCC-BY-NC 4.0 International license.

\begin{tabular}{|c|c|c|c|c|c|c|c|c|}
\hline Package & $\begin{array}{l}\text { Method } \\
\text { Key Words }\end{array}$ & $\begin{array}{l}\text { Method } \\
\text { Type }\end{array}$ & Reference & Availability & Language & Replicates & $\begin{array}{l}\text { Missing } \\
\text { Values }\end{array}$ & $\begin{array}{c}\text { Uneven } \\
\text { Sampling }\end{array}$ \\
\hline $\begin{array}{l}\text { Lomb-Scargle } \\
\text { (LS) }\end{array}$ & Periodogram & Parametric & $\begin{array}{l}\text { Bioinformatics } \\
\text { (2006) }\end{array}$ & $\frac{\underline{\text { https://www.iiap.res.in/astrostat/tuts/Lomb-Sc }}}{\underline{\text { argle.html }}}$ & $\mathrm{R}$ & $\checkmark$ & $\mathcal{V}$ & $\mathcal{V}$ \\
\hline $\begin{array}{l}\text { ARSER } \\
(\text { ARS })\end{array}$ & $\begin{array}{l}\text { Harmonic } \\
\text { Regression }\end{array}$ & Parametric & $\begin{array}{l}\text { Bioinformatics } \\
\text { (2010) }\end{array}$ & $\underline{\text { http://bioinformatics.cau.edu.cn/ARSER }}$ & Python \& R & $x$ & $x$ & $x$ \\
\hline $\begin{array}{l}\text { JTK_CYCLE } \\
\text { (JTK) }\end{array}$ & $\begin{array}{l}\text { Kendall's } \\
\text { Tau }\end{array}$ & $\begin{array}{l}\text { Non- } \\
\text { parametric }\end{array}$ & $\begin{array}{l}\text { J Biol Rhythms } \\
\quad \text { (2010) }\end{array}$ & $\frac{\text { https://openwetware.org/wiki/HughesLab:JTK }}{\underline{\text { Cycle }}}$ & $\mathrm{R}$ & $\mathcal{V}$ & $\mathcal{V}$ & $x$ \\
\hline RAIN & $\begin{array}{l}\text { Asymmetric } \\
\text { waveforms }\end{array}$ & $\begin{array}{c}\text { Non- } \\
\text { parametric }\end{array}$ & $\begin{array}{l}J \text { Biol Rhythms } \\
\text { (2014) }\end{array}$ & $\underline{\text { http://bioconductor.org/packages/rain }}$ & $\mathrm{R}$ & $\checkmark$ & $\checkmark$ & $\checkmark$ \\
\hline $\begin{array}{l}\text { eJTK_CYCLE } \\
(\text { eJTK })\end{array}$ & $\begin{array}{l}\text { Empirical } \\
p \text {-values }\end{array}$ & $\begin{array}{l}\text { Non- } \\
\text { parametric }\end{array}$ & $\begin{array}{l}\text { PLOS Comp. Bio. } \\
\text { (2015) }\end{array}$ & $\frac{\text { https://github.com/alanlhutchison/empirical-J }}{\underline{\text { TK CYCLE-with-asymmetry }}}$ & Python & $\checkmark$ & $\checkmark$ & $\checkmark$ \\
\hline $\begin{array}{l}\text { MetaCycle } \\
\text { (MC) }\end{array}$ & Integration & Parametric & $\begin{array}{l}\text { Bioinformatics } \\
\quad \text { (2016) }\end{array}$ & $\underline{\text { https://cran.r-project.org/package=MetaCycle }}$ & $\mathrm{R}$ & $\checkmark$ & $\mathcal{V}$ & $\checkmark$ \\
\hline $\begin{array}{l}\text { BIO_CYCLE } \\
\text { (BC) }\end{array}$ & $\begin{array}{l}\text { Deep Neural } \\
\text { Network }\end{array}$ & Parametric & $\begin{array}{l}\text { Bioinformatics } \\
\text { (2016) }\end{array}$ & $\underline{\mathrm{http}: / / \text { circadiomics.igb.uci.edu }}$ & $\mathrm{R}$ & $\checkmark$ & $M / x^{a}$ & $\checkmark$ \\
\hline
\end{tabular}


bioRxiv preprint doi: https://doi.org/10.1101/2020.04.04.024729; this version posted May 15, 2020. The copyright holder for this preprint (which was not certified by peer review) is the author/funder, who has granted bioRxiv a license to display the preprint in perpetuity. It is made available under aCC-BY-NC 4.0 International license.

\begin{tabular}{|c|c|c|c|c|c|c|c|c|}
\hline Design & Name & Reference & $\begin{array}{c}\text { Accession } \\
\text { Number }\end{array}$ & $\begin{array}{c}\text { Tissue } \\
\text { Type }\end{array}$ & $\begin{array}{l}\text { Sequencing } \\
\text { Platform }\end{array}$ & $\begin{array}{c}\text { Number of Time } \\
\text { Points \& } \\
\text { Replicates }\end{array}$ & $\begin{array}{c}\text { Number of } \\
\text { Genes }\end{array}$ & Time Points \\
\hline \multirow{4}{*}{ (A) Dark-Dark } & Hughes et al. & $\begin{array}{c}\text { PLOS Genetics } \\
\text { (2009) }\end{array}$ & GSE11923 & Liver & Microarray & $48 \times 1$ & 13,029 & CT18, 19, 20, ., 65 \\
\hline & $\begin{array}{l}\text { Hughes et al. } \\
\text { (downsampled) }\end{array}$ & $\begin{array}{c}\text { PLOS Genetics } \\
\text { (2009) }\end{array}$ & GSE11923 & Liver & Microarray & $24 \times 1$ & 12,506 & CT18, 20, 22, ., 64 \\
\hline & Hughes et al. & $\begin{array}{c}\text { PLOS Genetics } \\
\text { (2012) }\end{array}$ & GSE30411 & Liver & Microarray & $24 \times 1$ & 14,413 & $\mathrm{CT} 0,2,4, \ldots, 46$ \\
\hline & Zhang et al. & $\begin{array}{l}P N A S \\
(2014)\end{array}$ & GSE54652 & Liver & Microarray & $24 \times 1$ & 20,307 & CT18, 20, 22, .., 64 \\
\hline \multirow{4}{*}{ (B) Light-Dark } & Fang et al. & $\begin{array}{c}\text { Cell } \\
(2014)\end{array}$ & GSE59486 & Liver & GRO-seq & $8 \times 1$ & 17,463 & $\mathrm{ZT} 1,4,7,10,13,16,19,22$ \\
\hline & Menet et al. & $\begin{array}{l}\text { eLIFE } \\
\text { (2012) }\end{array}$ & GSE36872 & Liver & Nascent-seq & $6 \times 2$ & 17,917 & $\mathrm{ZT} 0,4,8,12,16,20$ \\
\hline & Menet et al. & $\begin{array}{l}\text { eLIFE } \\
(2012)\end{array}$ & GSE36871 & Liver & RNA-seq & $6 \times 2$ & 17,222 & $\mathrm{ZT} 2,6,10,14,18,22$ \\
\hline & Yang et al. & $\begin{array}{l}P N A S \\
(2018)\end{array}$ & GSE109938 & Liver & XR-seq (TS) & $6 \times 2$ & 17,652 & $\mathrm{ZT} 0,4,8,12,16,20$ \\
\hline
\end{tabular}




\begin{tabular}{|c|c|c|}
\hline Methods & Pros & Cons \\
\hline LS & $\begin{array}{l}\text { Effective in handling missing values } \\
\text { - Not restricted by input data structure (i.e. can be applied to } \\
\text { datasets with replicates, uneven samplings, or missing values) }\end{array}$ & $\begin{array}{l}\text { - Rapid degradation in detectability when applied to datasets with } \\
\text { low sampling resolution } \\
\text { - U-shaped } p \text {-values distribution } \\
\text { - Sensitive to outliers }\end{array}$ \\
\hline ARSER & - High reproducibility & - Cannot handle replicates, uneven samplings, or missing values \\
\hline JTK_CYCLE & $\begin{array}{l}\text { - } \quad \text { High precision } \\
\text { - Robust to outliers }\end{array}$ & $\begin{array}{l}\text { - Incapable of detecting asymmetric waveforms } \\
\text { - } \quad \text {-shaped } p \text {-values distribution } \\
\text { - } \quad \text { High false negative rates } \\
\text { - } \quad \text { Low reproducibility }\end{array}$ \\
\hline RAIN & $\begin{array}{l}\text { - High recall } \\
\text { - } \quad \text { Effective in detecting asymmetric waveforms } \\
\text { - } \quad \text { Not restricted by input data structure }\end{array}$ & $\begin{array}{l}\text { - High false positive rates } \\
\text { - } \quad \text { U-shaped } p \text {-values distribution } \\
\text { Computationally intensive with increasing sampling resolution }\end{array}$ \\
\hline eJTK_CYCLE & $\begin{array}{l}\text { - Uniform distribution of nominal } p \text {-values } \\
\text { - Most effective in detecting asymmetric waveforms }\end{array}$ & $\begin{array}{l}\text { - Unable to test different periods simultaneously } \\
\text { - Inefficient in handling missing values } \\
\text { - Sensitive to high level of uneven samplings }\end{array}$ \\
\hline MetaCycle & $\begin{array}{l}\text { - High recall } \\
\text { - Not restricted by input data structure } \\
\text { Offset the disadvantages of one method with the other two } \\
\text { among LS, ARSER and JTK_CYCLE } \\
\text { - Directly return calling results from three perspective methods } \\
\text { and perform ensemble }\end{array}$ & $\begin{array}{l}\text { - } P \text {-values generated with Fisher's integration require } \\
\text { independence assumption }\end{array}$ \\
\hline BIO_CYCLE & $\begin{array}{l}\text { - Most effective in controlling for false positive rates } \\
\text { - Most robust to data with high noise, uneven samplings, and } \\
\text { low sampling resolutions. } \\
\text { - High precision } \\
\text { High computational efficiency with pre-trained model }\end{array}$ & $\begin{array}{l}\text { - Require extensive time to train the DNN model } \\
\text { - Handle missing values only if data have replicates and the } \\
\text { missingness only pertains to part of the replicates. } \\
\text { - Low reproducibility }\end{array}$ \\
\hline
\end{tabular}

Table 3 